\title{
SOME $q$-IDENTITIES ASSOCIATED WITH RAMANUJAN'S CONTINUED FRACTION
}

\author{
BHASKAR SRIVASTAVA
}

\begin{abstract}
A continued fraction $C(-q, q)$ is defined as a special case of a general continued fraction $F(a, b, c, \lambda, q)$, which we have considered earlier in a separate paper. This continued fraction is also a special case of Ramanujan's continued fraction. In this paper we have found some very interesting $q$-identities and some identities analogous to identities given by Ramanujan involving $G(-q, q)$ and $H(-q, q)$ and one identity which gives the square of a continued fraction.
\end{abstract}

\section{Introduction}

In an earlier paper [4] we considered the continued fraction $C(-q, q)$, which is a special case of a continued fraction

$$
\begin{aligned}
F(a, b, c, \lambda, q)= & 1+\frac{(1-1 / c)(a q+\lambda q)}{(1+a q / c)+} \frac{b q+\lambda q^{2}}{1+} \\
& \times \frac{(1-1 / c q)\left(a q^{2}+\lambda q^{3}\right)}{(1+a q / c)+} \frac{b q^{2}+\lambda q^{4}}{1+\cdots \cdots} \\
= & \frac{\sum_{n=0}^{\infty}(-\lambda / a)_{n}(c)_{n}(-a q / c)^{n} /(q)_{n}(-b q)_{n}}{\sum_{n=0}^{\infty}(-\lambda / a)_{n}(c)_{n}\left(-a q^{2} / c\right)^{n} /(q)_{n}(-b q)_{n}}
\end{aligned}
$$

which is a generalization of the continued fraction of Ramanujan [1]. In the limit this continued fraction gives most of the classical results in continued fractions. For $c \rightarrow \infty$, it reduces to the unusual continued fraction of Ramanujan, which Andrews discovered in the "Lost" manuscript of Ramanujan [2]. By taking $a=0, b=0, \lambda=1$ and $c=-q$ in (1.1), we have the continued fraction $C(-q, q)$.

1991 Mathematics Subject Classification: 33D

Key words and phrases: continued fraction, basic hypergeometric series

Received January 24, 2000; revised July 25, 2000. 


$$
\begin{aligned}
C(-q, q) & =1+\frac{(1+1 / q) q}{1+} \frac{q^{2}}{1+} \frac{\left(1+1 / q^{2}\right) q^{2}}{1+} \frac{q^{4}}{1+\cdots \cdots} \\
& =1+\frac{\sum_{n=0}^{\infty} q^{n(n-1) / 2}(-q)_{n} /(q)_{n}}{\sum_{n=0}^{\infty} q^{n(n+1) / 2}(-q)_{n} /(q)_{n}} \\
& =1+\frac{\left(q^{2} ; q^{4}\right)^{2}}{\left(q^{3} ; q^{4}\right)_{\infty}\left(q ; q^{4}\right)_{\infty}} \quad\left(\begin{array}{l}
\text { by using summation formula Slater } \\
{\left[\begin{array}{l}
3, \text { eqn } 8 \text { and 13] } \\
\text { Also }[4, \text { p. 200, eqn. 2.2 }]
\end{array}\right.}
\end{array}\right)
\end{aligned}
$$

\section{Notation}

$$
\begin{aligned}
\left(a ; q^{k}\right)_{n} & =\prod_{j=0}^{n-1}\left(1-a q^{k j}\right) ; \quad n \geq 1 \\
\left(a ; q^{k}\right)_{0} & =1 \\
\left(a ; q^{k}\right)_{\infty} & =\prod_{j \geq 0}\left(1-a q^{k j}\right),
\end{aligned}
$$

when $k=1, q^{k}$ shall be omitted from the various symbols, in case there is no chance of ambiguity.

\section{An interesting $q$-identity}

We shall prove the identity

$$
\begin{aligned}
& {\left[\frac{(1+1 / q) q}{1+} \frac{q^{2}}{1+} \frac{\left(1+1 / q^{2}\right) q^{3}}{1+} \frac{q^{4}}{1+\cdots \cdots \cdots}\right]^{2}} \\
& \quad=\frac{\left(q^{2} ; q^{4}\right)_{\infty}^{2}}{\left(q^{4} ; q^{4}\right)_{\infty}^{2}}\left[\sum_{n=0}^{\infty} q^{4 n^{2}+2 n} \frac{1+q^{4 n+1}}{1-q^{4 n+1}}-\sum_{n=0}^{\infty} q^{4 n^{2}+6 n+2} \frac{1+q^{4 n+3}}{1-q^{4 n+3}}\right]
\end{aligned}
$$

The proof of this identity depends on Ramanujan's ${ }_{1} \Psi_{1}$-summation [1, p. 101], namely

$$
\begin{aligned}
{ }_{1} \Psi_{1}[a ; b ; q, z] & =\sum_{n=-\infty}^{\infty}(a)_{n} z^{n} /(b)_{n} \\
& =\frac{(b / a)_{\infty}(a z)_{\infty}(q / a z)_{\infty}(q)_{\infty}}{(q / a)_{\infty}(b / a z)_{\infty}(b)_{\infty}(z)_{\infty}}
\end{aligned}
$$

We shall first prove a series of identity: 


$$
\sum_{n=0}^{\infty} \frac{q^{i n}}{1-q^{4 n+i}}=\sum_{n=0}^{\infty} q^{4 n^{2}+2 i n} \frac{1+q^{4 n+i}}{1-q^{4 n+i}}, \quad i=1,2,3 .
$$

Now

$$
\begin{aligned}
\sum_{n=0}^{\infty} \frac{q^{i n}}{1-q^{4 n+i}} & =\sum_{n=0}^{\infty} \sum_{m=0}^{\infty} q^{i n+4 n m+i m} \\
& =\sum_{m=0}^{\infty} \sum_{n=0}^{\infty} q^{i(n+m)+4(n+m) m+i m}+\sum_{n=0}^{\infty} \sum_{m=0}^{\infty} q^{i n+4 n(m+n+1)+i(m+n+1)} \\
& =\sum_{m=0}^{\infty} \frac{q^{4 m^{2}+2 i m}}{1-q^{4 m+i}}+\sum_{n=0}^{\infty} \frac{q^{4 n^{2}+4 n+2 i n+i}}{1-q^{4 n+i}} \\
& =\sum_{n=0}^{\infty} q^{4 n^{2}+2 i n} \frac{1+q^{4 n+i}}{1-q^{4 n+i}}
\end{aligned}
$$

This proves (3.3).

By replacing $q$ by $q^{4}$ and then setting $a=q^{i}, b=q^{4+i}, z=q^{i}$ in (3.2), we have for $i=1,3$.

$$
\begin{aligned}
\sum_{n=-\infty}^{\infty} \frac{q^{i n}}{1-q^{4 n+i}} & =\frac{1}{1-q^{i}}{ }_{1} \Psi_{1}\left(q^{i} ; q^{4+i} ; q^{4}, q^{i}\right) \\
& =\frac{\left(q^{4} ; q^{4}\right)_{\infty}^{2}\left(q^{2 i} ; q^{4}\right)_{\infty}\left(q^{4-2 i} ; q^{4}\right)_{\infty}}{\left(q^{4-i} ; q^{4}\right)_{\infty}^{2}\left(q^{i} ; q^{4}\right)_{\infty}^{2}}
\end{aligned}
$$

Hence

$$
\begin{aligned}
\sum_{n=0}^{\infty} q^{4 n^{2}+2 n} \frac{1+q^{4 n+1}}{1-q^{4 n+1}}-\sum_{n=0}^{\infty} q^{4 n^{2}+6 n+2} \frac{1+q^{4 n+3}}{1-q^{4 n+3}} \\
=\sum_{n=0}^{\infty} \frac{q^{n}}{1-q^{4 n+1}}-\sum_{n=0}^{\infty} \frac{q^{3 n+2}}{1-q^{4 n+3}}, \quad \text { by }(3.3) \\
=\sum_{n=-\infty}^{\infty} \frac{q^{n}}{1-q^{4 n+1}} \\
=\frac{\left(q^{4} ; q^{4}\right)_{\infty}^{2}\left(q^{2} ; q^{4}\right)_{\infty}^{2}}{\left(q^{3} ; q^{4}\right)_{\infty}^{2}\left(q ; q^{4}\right)_{\infty}^{2}}, \quad \text { by }(3.4) \\
=\frac{\left(q^{4} ; q^{4}\right)_{\infty}^{2}\left(q^{2} ; q^{4}\right)_{\infty}^{4}}{\left(q^{2} ; q^{4}\right)_{\infty}^{2}\left(q^{3} ; q^{4}\right)_{\infty}^{2}\left(q ; q^{4}\right)_{\infty}^{2}} \\
\left.=\frac{\left(q^{4} ; q^{4}\right)_{\infty}^{2}[(1+1 / q) q}{\left(q^{2} ; q^{4}\right)_{\infty}^{2}} \frac{q^{2}}{1+} \frac{\left(1+1 / q^{2}\right) q^{3}}{1+} \frac{q^{4}}{1+\cdots \cdots}\right]^{2} .
\end{aligned}
$$


This proves (3.1).

\section{Some more identities}

Let us define

$$
\begin{aligned}
G(-q, q) & =\sum_{n=0}^{\infty} q^{n(n-1) / 2}(-q)_{n} /(q)_{n} \\
& =(-q, q)_{\infty}\left[\frac{1}{\left(q^{2} ; q^{4}\right)_{\infty}}+\frac{\left(q^{2} ; q^{4}\right)_{\infty}}{\left(q ; q^{4}\right)_{\infty}\left(q^{3} ; q^{4}\right)_{\infty}}\right],
\end{aligned}
$$

using summation formula Slater [3, eqn. 3] and

$$
\begin{aligned}
H(-q, q) & =\sum_{n=0}^{\infty} q^{n(n+1) / 2}(-q)_{n} /(q)_{n} \\
& =\frac{(-q ; q)_{\infty}}{\left(q^{2} ; q^{4}\right)_{\infty}}
\end{aligned}
$$

using summation formula Slater [3, eqn. 8].

Obviously

$$
C(-q, q)=\frac{G(-q, q)}{H(-q ; q)}
$$

and

$$
G(-q, q)=H(-q, q)+\frac{(-q ; q)_{\infty}\left(q^{2} ; q^{4}\right)_{\infty}}{\left(q ; q^{4}\right)_{\infty}\left(q^{3} ; q^{4}\right)_{\infty}} .
$$

Next we give a generalization of (3.4), namely

$$
\sum_{n=-\infty}^{\infty} \frac{q^{i n}}{1-q^{4 n+j}}=\frac{\left(q^{4} ; q^{4}\right)_{\infty}^{2}\left(q^{i+j} ; q^{4}\right)_{\infty}\left(q^{4-i-j} ; q^{4}\right)_{\infty}}{\left(q^{j} ; q^{4}\right)_{\infty}\left(q^{4-j} ; q^{4}\right)_{\infty}\left(q^{i} ; q^{4}\right)_{\infty}\left(q^{4-i} ; q^{4}\right)_{\infty}}
$$

where $0<i \leq 3,0<j \leq 3$ and $i+j \neq 4$.

The proof is similar to that of (3.4).

With the help of (4.1), (4.2), (4.4) and (4.5) we have the following identities, which are analogous to the identities of Ramanujan [2, pp. 197-198]

$$
\frac{\left(q^{4} ; q^{4}\right)_{\infty}^{2}}{(-q ; q)_{\infty}^{2}}[H(-q, q)]^{2}=\sum_{n=-\infty}^{\infty} \frac{q^{n}}{1-q^{4 n+2}}, \quad(i=1, j=2 \text { in }(4.5))
$$

$$
\frac{\left(q^{4} ; q^{4}\right)_{\infty}^{2}}{(-q ; q)_{\infty}^{2}}[G(-q ; q)-H(-q ; q)]^{2}=\sum_{n=-\infty}^{\infty} \frac{q^{n}}{1-q^{4 n+1}}, \quad(i=1, j=1 \text { in }
$$




$$
\begin{aligned}
& \frac{\left(q^{4} ; q^{4}\right)_{\infty}^{2}}{(-q ; q)_{\infty}^{2}}[H(-q, q)]^{2}=\sum_{n=-\infty}^{\infty} \frac{q^{2 n}}{1-q^{4 n+1}}, \quad(i=2, j=1 \text { in } \\
& \frac{\left(q^{4} ; q^{4}\right)_{\infty}^{2}}{(-q ; q)_{\infty}^{2}}\left[G(-q, q)-(H(-q, q)]^{2}=\sum_{n=-\infty}^{\infty} q^{2 n(2 n+1)} \frac{1+q^{4 n+1}}{1-q^{4 n+1}},\right.
\end{aligned}
$$

(Using (4.7) and and putting in for $i=1$ )

\section{Another continued fraction of the rogers-ramanujan type}

By making $q \rightarrow q^{2}$ and then putting $a=0, b=0, c=q^{2}$ and $\lambda=(1+1 / q)$ in (1.1), we have

$$
\begin{aligned}
\frac{\left(1-1 / q^{2}\right)\left(q+q^{2}\right)}{1+} \frac{q^{3}+q^{4}}{1+} \frac{\left(1-1 / q^{4}\right)\left(q^{5}+q^{6}\right)}{1+\cdots \cdots} & \\
= & \frac{\sum_{n=0}^{\infty}(-1)^{n}(1+1 / q)^{n} q^{n^{2}+n}}{\sum_{n=0}^{\infty}(-1)^{n}(1+1 / q)^{n} q^{n^{2}-n}}
\end{aligned}
$$

Let

$$
\frac{\sum_{n=0}^{\infty}(-1)^{n}(1+1 / q)^{n} q^{n^{2}+n}}{\sum_{n=0}^{\infty}(-1)^{n}(1+1 / q)^{n} q^{n^{2}-n}}=\sum_{n=0}^{\infty} a_{n} q^{n},
$$

Thus

$$
\begin{aligned}
1-(1+ & 1 / q) q^{2}+(1+1 / q)^{2} q^{6}-(1+1 / q)^{3} q^{12}+\cdots \cdots \cdots \cdot \cdots \\
= & \left(a_{0}+a_{1} q+a_{2} q^{2}+\cdots \cdots\right) \\
& \times\left[1-(1+1 / q)+(1+1 / q)^{2} q^{2}-(1+1 / q)^{3} q^{6} \cdots \cdots \cdot \cdots\right]
\end{aligned}
$$

or

$$
\begin{aligned}
q-q^{2}- & q^{3}+q^{5}+2 q^{6}+q^{7}-q^{10}-3 q^{11}-3 q^{12}-q^{13} \cdots \cdots \cdots \\
= & \left(a_{0}+a_{1} q+a_{2} q^{2}+\cdots \cdots\right) \\
& \quad \times\left(-1+q+2 q^{2}+q^{3}-q^{4}-3 q^{5}-3 q^{6}-q^{7}+\cdots \cdots \cdots\right)
\end{aligned}
$$

Comparing the coefficients of the various powers of $q$, we get

$$
\sum_{n=1}^{\infty} a_{n} q^{n}=-q-q^{3}-2 q^{4}-4 q^{5}-8 q^{6}-\cdots \cdots \cdots \cdots
$$


Hence

$$
\begin{aligned}
& \frac{\left(1-1 / q^{2}\right)\left(q+q^{2}\right)}{1+} \frac{q^{3}+q^{4}}{1+} \frac{\left(1-1 / q^{4}\right)\left(q^{5}+q^{6}\right)}{1+\cdots \cdots} \\
& =-q-q^{3}-2 q^{4}-4 q^{5}-8 q^{6}-\cdots \cdots \cdots \cdots
\end{aligned}
$$

This continued fraction $C(-q, q)$ is also a special case of Ramanujan's work (Entry 9 and 13 in chapter 16 of Ramanujan's Second Note Book Memoirs of the AMS 53 (1985) No. 315).

The present paper was motivated by Andrews' treatment of the RogersRamanujan continued fraction $[1,2]$ and the technique employed in the proof is a straight forward modification of his technique.

\section{REFERENCES}

[1] G. E. Andrews, An introduction to Ramanujan's "lost" notebook, Amer. Math. Monthly, 86 (1979), 89-108.

[2 ] G. E. ANDrews, Ramanujan's "lost" notebook III the Rogers-Ramanujan continued fraction, Adv. in Math., 41 (1981), 186-208.

[ 3 ] L. J. Slater, Further identities of the Rogers-Ramanujan type, Proc. London Math. Soc. (2), 54 (1952), 147-167.

[4] Bhaskar Srivastava, A continued fraction and some identities associated with Ramanujan, Riv. Mat. Univ. Parma (4), 17 (1991), 199-206.

Department of Mathematics

LUCKNOW UNIVERSITY

LUCKNOW, INDIA. 